Proceedings of the 2009 Winter Simulation Conference

M. D. Rossetti, R. R. Hill, B. Johansson, A. Dunkin, and R. G. Ingalls, eds.

\title{
EFFICIENT RARE EVENT SIMULATION OF CONTINUOUS TIME MARKOVIAN PERPETUITIES
}

\author{
Jose Blanchet \\ Department of Industrial Engineering and Operations Research \\ 500 W 120th St. 340 \\ New York, NY. 10025 \\ Peter Glynn \\ Department of Management Science and Engineering \\ Terman Eng. Building 313 \\ Stanford, CA. 94305
}

\begin{abstract}
We develop rare event simulation methodology for the tail of a perpetuity driven by a continuous time Markov chain. We present a state-dependent importance sampling estimator in continuous time that can be shown to be asymptotically optimal in the context of small interest rates.
\end{abstract}

\section{INTRODUCTION}

We concentrate on developing an efficient rare event simulation estimator for the tail distribution of a perpetuity (also known as infinite horizon discounted reward) in which both the discount rate and the reward rate are stochastic and driven by a continuous time Markov chain.

The distribution of a perpetuity arises in many applied settings including risk theory, mathematical finance, communication networks, number theory and computer science (see, for example, Vervaat (1979), Embrechts and Goldie (1994), Goldie and Grubel (1996) and Maulik and Zwart (2006)). In the setting of risk theory and finance it is natural to study perpetuities that evolve in a stochastic economic environment. Such has been the topic of a substantial number papers in the literature. The article of Paulsen (1998) surveys the literature on the analysis of perpetuities in the context of risk theory and finance. Most of the results focus on the development of asymptotic approximations for the tail distribution of a perpetuity or closed formulae in some classes of models. More recent articles on this type of analysis include Nyrhinen (2001) and Kluppelberg and Kostadinova (2008). The text of Asmussen (2001) also includes a section on the analysis of perpetuities and their role in risk theory.

Most of the simulation work on perpetuities concentrate on the design of algorithms to generate unbiased samples and this is primarily in the discrete case and in the context of independent and identically distributed (i.i.d.) rewards and discounts (see, for instance, Devroye, Fill, and Neininger (2000) and Devroye, and Neininger, (2002)). Rare event simulation for perpetuities, on the other hand has been studied in Asmussen and Nielsen (1995) in the context of deterministic interest rates and Blanchet and Zwart (2007) in some discrete settings involving i.i.d. discount rates. In contrast, we concentrate on perpetuities in continuous time driven by Markov chains.

Our analysis here concerns an environment involving small discount rates, which is motivated by the situation that governs "safe instruments" such as the treasury bonds these days. These types of asymptotic environments have not been well studied in the literature in the context of risk theory and simulation (see Blanchet (2004) for a related type of analysis). Since insurance companies tend to invest (because of regulation) in safe instruments we consider that our considerations here are of significant relevance.

We assume that the discount rate $(\gamma(x): x \in S)$ is positive and the reward rate $(\lambda(x): x \in S)$ are governed by an irreducible continuous time Markov chain $(X(t): t \geq 0)$ taking values on a finite state-space $S$. We concentrate on the finite state-space case for simplicity, but the analysis in the context of more general processes will appear elsewhere and is similar to that of Blanchet and Glynn (2009); see also Blanchet (2004). 
The perpetuity $D(\delta)$ then takes the form

$$
D(\delta)=\int_{0}^{\infty} \exp \left(-\delta \int_{0}^{t} \gamma(X(s)) d s\right) \lambda(X(t)) d t
$$

For notational convenience we introduce the cumulative discount and reward processes $\Gamma(t)=\int_{0}^{t} \gamma(X(s)) d s$ and $\Lambda(t)=$ $\int_{0}^{t} \lambda(X(s)) d s$ respectively, so that $D(\delta)=\int_{0}^{\infty} \exp (-\delta \Gamma(t)) d \Lambda(t)$. Moreover, note that ergodic theory guarantees the existence of constants $\bar{\gamma}>0$ and $\bar{\lambda}$ such that $\Gamma(t)=\bar{\gamma} t+o(t)$ and $\Lambda(t)=\bar{\lambda} t+o(t)$ as $t \nearrow \infty$ (where $o(t) / t \longrightarrow 0$ almost surely as $t \nearrow \infty)$. In addition, it follows easily from the previous asymptotic relation for $\Gamma(\cdot)$ that $\Gamma^{-1}(t)=t / \bar{\gamma}+o(t)$ almost surely as $t \nearrow \infty$.

Now, integrating by parts yields that

$$
\begin{aligned}
D(\delta) & =\int_{0}^{\infty} \exp (-\delta \Gamma(t)) d \Lambda(t) \\
& =\delta \int_{0}^{\infty} \Lambda(t) \exp (-\delta \Gamma(t)) d \Gamma(t)
\end{aligned}
$$

Making the change-of-variable $\delta \Gamma(t)=u$ we obtain

$$
D(\delta)=\int_{0}^{\infty} \exp (-u) \Lambda\left(\Gamma^{-1}(u / \delta)\right) d u
$$

Taking advantage of the asymptotic relations explained in the previous paragraph it follows immediately that $\delta D(\delta) \longrightarrow \bar{\lambda} / \bar{\gamma}$ almost surely as $\delta \searrow 0$. Our interest is in large deviations from the "typical" value $\bar{\lambda} /(\delta \bar{\gamma})$ of $D(\delta)$, so we concentrated on estimating

$$
\alpha(\delta)=P(\delta D(\delta)>\eta)
$$

for $\eta \in\left(\bar{\lambda} / \bar{\gamma}, d_{*}\right)$, where $d_{*}=\max _{x \in S}|\lambda(x)| / \min _{x \in S} \gamma(x)<\infty$ (i.e. $d^{*}$ is the supremum of the support of $\delta D(\delta)$ ). Our goal is then to construct an importance sampling estimator whose relative mean squared error can be shown to be well controlled as $\delta \searrow 0$. In particular, we will exhibit an unbiased estimator whose coefficient of variation grows at most at a rate $o\left(\alpha(\delta)^{-\varepsilon}\right)$ for any $\varepsilon>0$ as $\delta \searrow 0$. (We use the notation $f(x)=o(x)$ if $f(x) / x \longrightarrow 0$ as $x \nearrow \infty$ ). An estimator that satisfies such a mean squared error criterion is said to be asymptotically optimal (see Asmussen and Glynn (2007) p. 159).

Section 2 presents the large deviations analysis for the perpetuity of interest. The construction of our importance sampling estimator and the efficiency analysis is given in Section 3.

\section{PRELIMINARIES ON MARKOV PROCESSES AND LARGE DEVIATIONS FOR PERPETUITIES}

Equation (1) represents $\delta D(\delta)$ as a linear functional of the process $\delta Z(\cdot / \delta) \triangleq \delta \Lambda\left(\Gamma^{-1}(\cdot / \delta)\right)$; namely

$$
\delta D(\delta)=F(\delta Z(\cdot) / \delta) \triangleq \int_{0}^{\infty} \exp (-u) \delta Z(u / \delta) d u
$$

In turn, sample paths large deviations for $\delta Z(\cdot / \delta)$ can be developed under a suitable topology under which $F(\cdot)$ is continuous. This approach, which provides a representation for the rate function of $\delta D(\delta)$ is studied in detail in Blanchet (2004) (see also Blanchet and Glynn (2009)). Here we concentrate instead on obtaining, from first principles, the asymptotic log-moment generating function of $D(\delta)$. This will allow us to understand the nature of the importance sampling strategy that we shall implement.

Large deviations estimates for Markov additive processes, such as $\Gamma(\cdot)$ and $\Lambda(\cdot)$, require the specification of suitable eigenvalue and eigen-functions. Given the connection described in the previous paragraph it is not surprising that such types of quantities are also required to obtain the asymptotic log-moment generating function of $D(\delta)$. We shall work directly with the process $Z(\cdot)=\Lambda\left(\Gamma^{-1}(\cdot)\right)$. We note that $\Gamma^{-1}(\cdot)$ induces a random time change and that the process $Y(\cdot)$ defined 


\section{Blanchet and Glynn}

via $Y(t)=X\left(\Gamma^{-1}(t)\right)$ is also a continuous time Markov chain with intensity matrix (or generator) $A^{Y}$ defined via

$$
\left(A^{Y} v\right)(y)=\frac{(A v)(x)}{\gamma(x)}
$$

We use $v(\cdot)$ to denote a generic vector, $v(x)$ is the entry corresponding to state $x$. The validity of (3) can be easily verified from first principles. In turn,

$$
\begin{aligned}
Z(t) & =\Lambda\left(\Gamma^{-1}(t)\right)=\int_{0}^{\Gamma^{-1}(t)} \lambda(X(s)) d s \\
& =\int_{0}^{t} \frac{\lambda\left(X\left(\Gamma^{-1}(u)\right)\right)}{\gamma\left(X\left(\Gamma^{-1}(u)\right)\right)} d u \triangleq \int_{0}^{t} \beta(Y(u)) d u
\end{aligned}
$$

where $\lambda(y) / \gamma(y)=\beta(y)$ is also a Markov additive process with respect to $Y$. The Perron-Frobenius theory (see Asmussen (2003) p. 25) implies that for each $\theta \in(-\infty, \infty)$ there exists a positive eigenvalue $\psi(\theta)$ and a positive eigenvector $u(\cdot, \theta)$ such that

$$
\left(A_{Y} u\right)(y, \theta)=(\psi(\theta)-\beta(y)) u(y, \theta) .
$$

Now, given $r>0$, consider the space-time Markov chain $W(\cdot)$ defined via

$$
W(t)=(Y(t), r+t) .
$$

It is easy to verify that the generator of $W(\cdot)$ can formally be written as $A_{W}=A_{Y}+\partial_{t}$. More precisely, given $(g(y, t): y \in S, t \geq 0)$ we have that $\left(A_{W} g\right)(y, r+t)=\left(A_{Y} g\right)(y, r+t)+\partial_{t} g(y, r+t)$. The matrix $A_{Y}$ is applied to the vector $g(\cdot, r+t)$ and $\partial_{t}$ represent the derivative with respect to $t$ applied to the function $g(y, r+\cdot)$. We hereafter use the notation $\left(A_{Y}+\partial_{t}\right) g(y, r+t)$ to describe $\left(A_{W} g\right)(y, r+t)$.

The following result is very useful to construct simulation algorithms for Markov processes (cf. Skorokhod, Hoppensteadt, and Salehi (2002) p. 82)

Lemma 1. Given a positive function $(h(y, t): y \in S, t \geq 0)$ which belongs to the generator of $A_{W}$ we have that the process

$$
M_{h}(t)=h(Y(t), t+r) \exp \left(\int_{0}^{t}\left(\frac{\left(A_{Y}+\partial_{s}\right) h(Y(s), r+s)}{h(Y(s), r+s)}\right) d s\right)
$$

is a martingale.

Proof. The expression for $M_{h}(t)$ is equivalent to

$$
M_{h}(t)=h(W(t)) \exp \left(-\int_{0}^{t}\left(\frac{\left(A_{W} h\right)(W(s))}{h(W(s))}\right) d s\right) .
$$

Recall that if $h(\cdot)$ is in the domain of $A_{W}$ then $N(\cdot)$ defined via

$$
N_{h}(t)=h(W(t))-\int_{0}^{t}\left(A_{W} h\right)(W(s)) d s
$$




\section{Blanchet and Glynn}

is a martingale (in fact it is known as the Dynkin martingale). We then apply the product rule of differentiation of standard calculus to $M_{h}(t)$ (everything here is of bounded variation) to obtain

$$
\begin{aligned}
d M_{h}(t)= & -M_{h}(t) \frac{\left(A_{W} h\right)(W(t))}{h(W(t))} d t \\
& +\frac{M_{h}(t)}{h(W(t))} d\left(N_{h}(t)+\int_{0}^{t}\left(A_{W} h\right)(W(s)) d s\right) \\
= & \frac{M_{h}(t)}{h(W(t))} d N_{h}(t) .
\end{aligned}
$$

Since $N_{h}(\cdot)$ is a martingale and the integrand is bounded we conclude that $M_{h}(\cdot)$ is a martingale.

The previous lemma indicates that $M_{h}(\cdot)$ is a positive martingale, therefore, $M_{h}(\cdot) / h(W(0))$ induces a change-of-measure whose associated probability in the path space of $W(\cdot)$ will be denoted by $P^{(h)}$. It follows from Lemma 3 in p. 82 of Skorokhod, Hoppensteadt, and Salehi (2002) that under $P^{(h)}$ the process $W(\cdot)$ is also a Markovian with infinitesimal generator $A_{W}^{(h)}$ given by

$$
\left(A_{W}^{(h)} g\right)(w)=\frac{\left(A_{W} g \otimes h\right)(w)-g(w)\left(A_{W} h\right)(w)}{h(w)} .
$$

The notation $g \otimes h$ represents a tensor product. In other words $(g \otimes h)(w)=g(w) h(w)=g(y, t) h(w, t)$.

We now are ready to obtain an expression for the asymptotic log-moment generating function of $D(\delta)$. The idea is to judiciously select the function $h(\cdot)$ in order to construct a martingale such as $M_{h}(\cdot)$. We define

$$
h(y, t)=u(y, \theta \exp (-\delta t))
$$

where $u(\cdot)$ is selected so that $u(y, 0)=1$. Observe, using (4), that

$$
\begin{aligned}
\left(A_{W} h\right)(y, t) & =\left(A_{Y} u\right)(y, \theta \exp (-t)) \\
& +\theta \delta \partial_{\theta} u(y, \theta \exp (-\delta t)) \exp (-\delta t) \\
& =(\psi(\theta \exp (-\delta t))-\theta \exp (-\delta t) \beta(y)) u(y, \theta \exp (-\delta t)) \\
& +\theta \delta \partial_{\theta} u(y, \theta \exp (-\delta t)) \exp (-\delta t) .
\end{aligned}
$$

Consequently,

$$
\begin{aligned}
M_{h}(t) & =u(Y(t), \theta \exp (-t)) \\
& \times \exp \left(\theta \int_{0}^{t} \exp (-\delta s) \beta(Y(s)) d s-\int_{0}^{t} \psi(\theta \exp (-\delta s)) d s\right) \\
& \times \exp \left(\theta \delta \int_{0}^{t} \frac{\partial_{\theta} u(Y(s), \theta \exp (-\delta s))}{u(Y(s), \theta \exp (-\delta s))} \exp (-\delta s) d s\right)
\end{aligned}
$$

Clearly $\left(M_{h}(t): t \geq 0\right)$ is uniformly bounded and therefore uniformly integrable. Therefore, we can send $t \nearrow \infty$ obtaining convergence almost surely and in $L_{1}$ to

$$
\begin{aligned}
M_{h}(\infty) & =\exp \left(\theta \int_{0}^{\infty} \exp (-\delta s) \beta(Y(s)) d s-\int_{0}^{\infty} \psi(\theta \exp (-\delta s)) d s\right) \\
& \times \exp \left(\theta \delta \int_{0}^{\infty} \frac{\partial_{\theta} u(Y(s), \theta \exp (-\delta s))}{u(Y(s), \theta \exp (-\delta s))} \exp (-\delta s) d s\right)
\end{aligned}
$$




\section{Blanchet and Glynn}

Finally, observe (given that the function $\partial_{\theta} u(y, \cdot)$ is continuous by results of Kontoyiannis and Meyn (2003)) that there exists a constant $\kappa \in(0, \infty)$ independent of $\delta$ such that

$$
\begin{aligned}
\kappa^{-1} & \leq \delta \int_{0}^{\infty} \frac{\partial_{\theta} u(Y(s), \theta \exp (-\delta s))}{u(Y(s), \theta \exp (-\delta s))} \exp (-\delta s) d s \\
& =\int_{0}^{\infty} \frac{\partial_{\theta} u(Y(u / \delta), \theta \exp (-u))}{u(Y(u / \delta), \theta \exp (-u))} \exp (-u) d u \leq \kappa
\end{aligned}
$$

Therefore, $\kappa \in(0, \infty)$ such that

$$
\begin{aligned}
& \exp \left(\kappa^{-1}\right) E \exp \left(\theta \int_{0}^{\infty} \exp (-\delta s) \beta(Y(s)) d s\right) \\
& \leq \exp \left(\int_{0}^{\infty} \psi(\theta \exp (-\delta s)) d s\right) E M_{h}(\infty) \\
& \leq \exp (\kappa) E \exp \left(\theta \int_{0}^{\infty} \exp (-\delta s) \beta(Y(s)) d s\right) .
\end{aligned}
$$

On the other hand, using the definition of $Z(s)$, integration by parts and equation (2) we obtain that

$$
D(\delta)=\int_{0}^{\infty} \exp (-\delta s) \beta(Y(s)) d s
$$

Consequently, we obtain the following result.

Theorem 1. Given that $Y(0)=x$

$$
\lim _{\delta \backslash 0} \delta \log E_{x}(\exp (\theta D(\delta)))=\int_{0}^{\infty} \psi(\theta \exp (-t)) d t
$$

Proof. Since $M_{h}(t)$ converges to $M_{h}(\infty)$ almost surely and in $L_{1}$ we have that $E_{x}\left(M_{h}(\infty)\right)=u(x, \theta)$. The result follows directly from (7) and equation inequality (8).

As a consequence of the previous result we obtain the following Theorem (see also Blanchet (2004) and Blanchet and Glynn (2009)).

Theorem 2. Given $\eta \in\left(\bar{\lambda} / \bar{\gamma}, d_{*}\right)$ we have that

$$
\alpha(\delta)=\exp (-I(\eta) / \delta+o(1 / \delta))
$$

as $\delta \searrow 0$ where

$$
I(\eta)=\psi\left(\theta^{*}\right)-\int_{0}^{\theta^{*}} \frac{\psi(r)}{r} d r
$$

and $\theta^{*} \triangleq \theta^{*}(\eta)$ satisfies

$$
\eta \theta^{*}(\eta)=\psi\left(\theta^{*}(\eta)\right) .
$$

Proof. The result follows by a direct application of the Gartner-Ellis theorem (see for instance Dembo and Zeitouni (1998) p. 43). To verify the validity of the expression for $I(\eta)$ note that for $\theta>0$

$$
\int_{0}^{\infty} \psi(\theta \exp (-t)) d t=\int_{0}^{\theta} \frac{\psi(r)}{r} d r .
$$




\section{Blanchet and Glynn}

Therefore, the associated Legendre transform of the asymptotic log-moment generating function of $D(\delta)$ evaluated at $\eta \in\left(\bar{\lambda} / \bar{\gamma}, d_{*}\right)$, namely, $I(\eta)$ satisfies

$$
I(\eta)=\max _{\theta \geq 0}\left(\eta \theta-\int_{0}^{\theta} \frac{\psi(r)}{r} d r\right) .
$$

Optimality conditions then yield that the optimum value $\theta^{*}(\eta)$ satisfies $\eta \theta^{*}(\eta)=\psi\left(\theta^{*}(\eta)\right)$, thereby obtaining the indicated expression for $I(\eta)$.

\section{IMPORTANCE SAMPLING ESTIMATOR AND EFFICIENCY}

As is often the case in rare-event simulation, specially in the context of light-tailed systems such as the ones that we consider here, the techniques behind the large deviations asymptotics provide useful guidance for the construction of efficient importance sampling estimators. Not surprisingly then, we will take advantage of the ideas and notions developed in the previous section to construct our estimator.

The construction of the estimator is a direct translation of the change-of-measure induced by the choice of $h(\cdot)$ indicated in (6) using $\theta=\theta^{*}$. More precisely, after having computed $\psi(\theta)$, we evaluate $\theta^{*}=\theta^{*}(\eta)$ satisfying the root equation indicated in Theorem 2. Then we write

$$
h_{*}(y, t)=u\left(y, \theta^{*} \exp (-\delta t)\right)
$$

We shall assume the initial condition $Y(0)=y_{0}$ (the sampler is explained in terms of the generator $A_{Y}$ ). We compute the rate matrix of the process $Y$ under $P_{y_{0}}^{\left(h_{*}\right)}$ using equation (5) with our specific choice of $h_{*}(\cdot)$ in place of $h(\cdot)$. In particular, we obtain that the rate matrix $A_{Y}^{\left(h_{*}\right)}$ of $Y$ under $P_{y_{0}}^{\left(h_{*}\right)}$ is time in-homogeneous given by

$$
\left(A_{Y}^{\left(h_{*}\right)} f\right)(y, t)=\frac{A_{Y}\left(f \otimes h_{*}\right)(y, t)-f(y)\left(A_{Y} h_{*}\right)(y, t)}{h_{*}(y, t)} .
$$

To obtain the column corresponding to state $y$ in the matrix $A_{Y}^{\left(h_{*}\right)}$ at a particular time $t$ we evaluate the right hand side of (9) selecting $f(\cdot)=e_{y}(\cdot)$, where $e_{y}(\cdot)=1$ if $y=x$ and 0 otherwise. This gives

$$
A_{Y}^{\left(h_{*}\right)}(x, y)=A_{Y}(x, y)-e_{x}(y) \frac{\left(A_{Y} h_{*}\right)(x, t)}{h_{*}(x, t)} .
$$

Simulation of time in-homogeneous Markov chains is standard and can be done using thinning (see Asmussen and Glynn (2007) p. 61).

After simulating the process $Y$ under the time in-homogeneous generator $A_{Y}^{\left(h_{*}\right)}$ we then compute the likelihood ratio

$$
\begin{aligned}
L(\delta) & \triangleq u\left(y_{0}, \theta^{*}\right) M_{h_{*}}^{-1}(\infty) \\
& =u\left(y_{0}, \theta^{*}\right) \exp \left(-\theta^{*}(D(\delta)-\eta / \delta)-I(\eta) / \delta\right) \\
& \times \exp \left(-\theta_{*} \delta \int_{0}^{\infty} \frac{\partial_{\theta} u\left(Y(s), \theta_{*} \exp (-\delta s)\right)}{u\left(Y(s), \theta_{*} \exp (-\delta s)\right)} \exp (-\delta s) d s\right) .
\end{aligned}
$$

The previous expression will certainly have to be truncated for implementation purposes thereby inducing a bias. Nevertheless, it is not difficult to convince oneself that such bias can be controlled by truncating at a level of order $\Omega\left(1 / \delta^{3 / 2}\right)$. We say that $f(x)=\Omega(x)$ if there exists $\kappa \in(0, \infty)$ such that $\kappa^{-1} x \leq f(x) \leq \kappa x$ for all $x$ larger than some $x_{0} \in(0, \infty)$.

We conclude with a summary of the efficiency properties of our estimator.

Theorem 3. The importance sampling estimator, $L(\delta) I(D(\delta)>\eta / \delta)$, is asymptotically optimal as $\delta \searrow 0$. 


\section{Blanchet and Glynn}

Proof. The mean squared error of the estimator is bounded by the square of its second moment (under the probability measure $P_{y_{0}}^{\left(h_{*}\right)}$, of course). So, using the inequality (7) and the expression for $L(\delta)$ defined in (10) we obtain that

$$
\begin{aligned}
& E_{y_{0}}^{\left(h_{*}\right)}\left(L(\delta)^{2} I(D(\delta)>\eta / \delta)\right) / \alpha(\delta)^{2} \\
\leq & \exp (2 \kappa) \exp (-2 I(\eta)) u\left(y_{0}, \theta^{*}\right) / \alpha(\delta)^{2} \\
& \times E_{y_{0}}^{\left(h_{*}\right)}\left(\exp \left(-2 \theta^{*}(D(\delta)-\eta / \delta)\right) I(D(\delta)>\eta / \delta)\right) \\
\leq & \exp (2 \kappa) u\left(y_{0}, \theta^{*}\right) \exp (o(1 / \delta)) .
\end{aligned}
$$

The previous line follows from Theorem 2, which also yields that the last expression in the previous display is $o\left(\alpha(\delta)^{-\varepsilon}\right)$ for any $\varepsilon>0$, thereby achieving asymptotic optimality.

\section{ACKNOWLEDGMENTS}

This research was partially supported by NSF grants NSF-0846816 and DMS-0806145.

\section{REFERENCES}

Asmussen, S. 2001. Ruin Probabilities. World Scientific. Singapore.

Asmussen, S. 2003. Applied Probability and Queues. Springer-Verlag. New York.

Asmussen, S. and P. Glynn. 2007. Stochastic Simulation: Algorithms and Analysis. Springer-Verlag. New York.

Asmussen, S. and H. M. Nielsen. 1995. Ruin probabilities via local adjustment coefficients. Journal of Applied Probability 32: $736-755$.

Blanchet, J. 2004. Approximations and Limit Theorems for Insurance Risk and Queueing Theory. Ph.D. Dissertation. Stanford University.

Blanchet, J. and P. Glynn. 2009 Large deviations and exact asymptotics for perpetuities. Preprint.

Blanchet, J., and B. Zwart. 2007 Importance sampling of compounding processes. Proceedings of the 2007 Winter Simulation Conference. eds. S. G. Henderson, B. Biller, M.-H. Hsieh, J. Shortle, J. D. Tew, and R. R. Barton, 372-379. Pistacaway, New Jersey: Institute of Electrical and Electronics Engineers, Inc.

Dembo, A., and O. Zeitouni. 1998. Large Deviations Techniques and Applications. Springer-Verlag. New York.

Devroye, L., J. Fill, and R. Neininger. 2000. Perfect simulation from the Quicksort limit distribution. Electronic Communications in Probability. 5, 95-99. (PDF file. Technical Report \#603, Department of Mathematical Sciences, The Johns Hopkins University)

Devroye, L., and R. Neininger. 2002. Density approximation and exact simulation of random variables that are solutions of fixed-point equations. Advances in Applied Probability. 24: 441-468.

Embrechts, P., and C. Goldie. 1994. Perpetuities and random equations. In: Mandl, P., Huskova, M. (eds.) Asymptotic Statistics. Proceedings of the 5th Prague Symposium, 75-86. Physica-Verlag.

Goldie, C. and R. Grübel. 1996. Perpetuities with thin tails. Advances in Applied Probability. 28: 463-480.

Kluppelberg, C. and R. Kostadinova. 2008. Integrated insurance risk models with exponential Levy investment. Insurance: Mathematics and Economics. 42: 560-577.

Kontoyiannis, I., and S. Meyn. 2003. Spectral theory and limit theorems for geometrically ergodic Markov processes. Annals of Applied Probability. 13: 304-362.

Maulik, K and B. Zwart. 2006. Tail asymptotics of exponential functionals of Lévy processes. Stochastic Processes and their Applications. 116: 156-177.

Nyrhinen, H. 2001. Finite and infinite time ruin probabilities in a stochastic economic environment. Stochastic Processes and their Applications. 92: 265-285.

Paulsen, J. 1998. Ruin theory with compounding assets - a survey. Insurance: Mathematics and Economics. 22 : 3-16.

Skorokhod, A., F. Hoppensteadt, and H. Salehi. 2002. Random Perturbation Methods with Applications to Science and Engineering. Springer-Verlag, New York.

Vervaat, W. 1979. On a stochastic difference equation and a representation of non-negative infinitely divisible random variables. Advances in Applied Probability. 11: 750-783. 


\section{Blanchet and Glynn}

\section{AUTHOR BIOGRAPHIES}

JOSE BLANCHET is Assistant Professor of IEOR at Columbia University. Jose holds a M.Sc. in Operations Research and a Ph.D. in Management Science and Engineering, both from Stanford University. Prior to joining Columbia he was a faculty member in the Statistics Department at Harvard University. Jose worked for two years as an analyst in Protego Financial Advisors, a leading investment bank in Mexico. He has research interests in applied probability, computational finance, performance engineering, queueing theory, risk management, rare-event analysis, statistical inference, stochastic modeling, and simulation. He serves or has served in the editorial board of the Applied Probability Journals, TOMACS and Statistica Sinica. His email is <jose.blanchetecolumbia.edu>

PETER W. GLYNN received his Ph.D. in Operations Research from Stanford University in 1982. He then joined the faculty of the University of Wisconsin at Madison, where he held a joint appointment between the Industrial Engineering Department and Mathematics Research Center, and courtesy appointments in Computer Science and Mathematics. In 1987, he returned to Stanford, where he is now the Thomas Ford Professor of Engineering in the Department of Management Science and Engineering. He also has a courtesy appointment in the Department of Electrical Engineering and serves as Director of the Stanford Institute of Computational and Mathematical Engineering. He is a member of INFORMS and a fellow of the Institute of Mathematical Statistics and his research interests in computational probability, simulation, queueing theory, statistical inference for stochastic processes, and stochastic modeling. His email is $\langle$ glynnestanford.edu> 